\title{
Verticalidad discursiva en la articulación entre organización no gubernamental, gobierno local y organizaciones sociales de base de mujeres: análisis desde la comunicación
}

\section{Resumen}

El presente estudio ofrece una explicación de la verticalidad discursiva, observada en la relación establecida entre una organización no gubernamental para el desarrollo (ONG), un gobierno local (GL) y organizaciones sociales de base (OSB) de apoyo alimentario conformadas por mujeres rurales; en el intento de "formar" ciudadanía. Se aborda el análisis desde una perspectiva dialéctica considerando que la verticalidad de tales relaciones es producto de situaciones históricas de exclusión y de la concentración desigual del poder. Se considera como marco de referencia el contexto socio-político de capitalismo dependiente, característico en algunos países de la región latinoamericana. El estudio pretende resaltar el papel fundamental que tiene la comunicación - aquella que se concibe como espacio de encuentro y liberación - para el cambio social, haciendo notar que su ausencia genera relaciones de verticalidad insostenibles, promoviendo cambios que no surgen de la conciencia y práctica de las personas y prolongan la reproducción del sistema desigual actual.

Palabras clave: verticalidad discursiva, mujeres rurales, exclusión, comunicación como liberación.

Vertical discourse in the communication between non-governmental organization, local government and women's community-based organizations: analysis from the communication perspective

\section{Abstract}

This study explains the vertical discourse observed in the relationship established between a non-
Eliana Pérez Barrenechea Universidad César Vallejo Recibido el 2 de diciembre de 2014 Aceptado el 20 de diciembre de 2014 
governmental organization for development (NGO), a local government (LG) and community-based organizations (CBO) for food support constituted by rural women in order to "create" citizenship.

The analysis is covered from the dialectic perspective taking into account that the vertical nature of such relations is the result of historical exclusion situations and the unequal concentration of power. Common in some Latin American countries, the social-political context of dependent capitalism is considered a reference framework.

The study intends to highlight the fundamental role of communication - conceived as a space of encounter and liberation - for social change. It is evidenced that the lack of communication generates unsustainable vertical relations, promoting changes that are not originated from the individual's consciousness and practice and extend the reproduction of the existing unequal system.

Keywords: vertical discourse, rural women, exclusion, communication as liberation.

\section{Introducción}

Este artículo ha sido elaborada en base al análisis crítico de los resultados obtenidos en la tesis realizada para optar el grado de maestra en Ciencias Sociales, que observaba las limitaciones discursivas en la promoción ciudadana por parte de una organización no gubernamental de desarrollo (ONG) y el gobierno local (GL), así como las condiciones de las mujeres de organizaciones sociales de base (OSB) de apoyo alimentario para la recepción del discurso. La ubicación del estudio es el distrito de Laredo, provincia de Trujillo, región La Libertad (Perú).

Los resultados revelan que existe una distancia cultural entre los emisores del discurso promotor de participación ciudadana (técnicos de la ONG y del GL) y las receptoras (mujeres de OSB), situación que no permite una horizontalidad comunicativa sino por el contrario, construye un discurso que, lejos de constituirse en una herramienta de cambio social, acentúa la ideología dominante de una seudodemocracia que no permite inclusión verdadera de los sectores excluidos y que reproduce las relaciones de poder entre autoridades, funcionarios, técnicos (quienes manejan y colocan 
el discurso) y las mujeres de base (quienes escuchan, asisten, "participan" en lo que se les pide).

En este estudio se describe un discurso cosmético de cambio social de la ONG y el discurso del GL que no evidencia prácticas efectivas de promoción ciudadana, limitado a cumplir con la exigencia normativa de hacer participar a la sociedad civil en espacios participativos obligatorios. Además, profundiza la explicación de las relaciones internas y externas que se producen en la dinámica discursiva de ambos emisores hacia las receptoras de la OSB.

El siguiente esquema sintetiza los aspectos que se abordan en este estudio. La verticalidad discursiva de la ONG y del Gobierno local con las mujeres de la OSB (verticalidad interna), pero también se indica que existe otra relación vertical entre los organismos de cooperación internacional (por sus políticas y condiciones impuestas) y las ONG locales, así como la hay entre las estructuras centralistas del Estado (que supeditan a las Municipalidades a decisiones político-económicas centrales) con los gobiernos locales. En ambos niveles de verticalidad (interna y externa) se producen respuestas adaptativas por parte de los emisores, que siguen reproduciendo verticalismo y por ende promoviendo una participación persuasivo-adaptativa que no engendra cambio social.

Figura 01: Modelo de la verticalidad discursiva

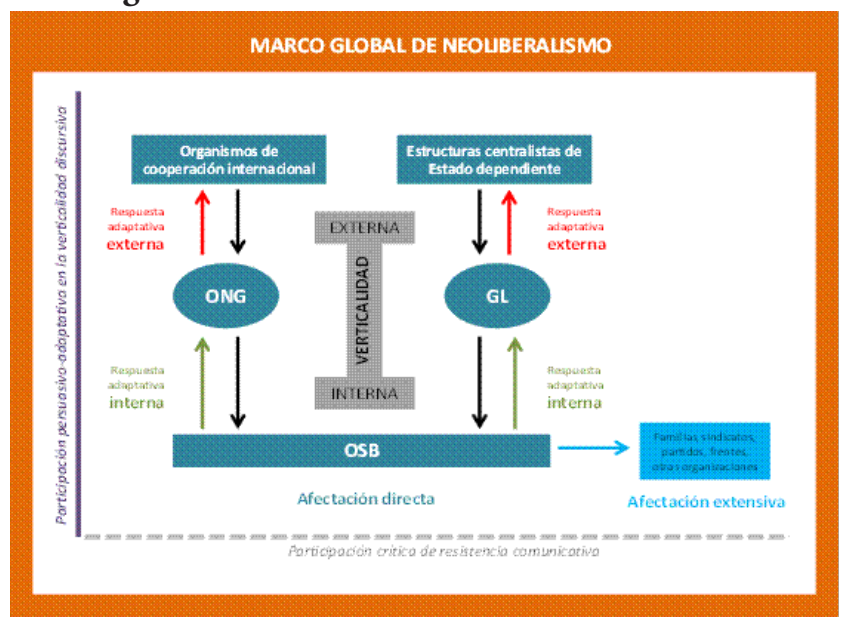

Fuente: Elaboración propia. 
Se agregan en el modelo -en una perspectiva de horizontalidad comunicativa - la respuesta que ha de producirse en un verdadero encuentro comunicativo, en una toma de conciencia propia, en un escenario de reflexión-acción elemental. En este caso habría de producirse una participación crítica de resistencia que rompa con la participación alegórica, vaciada de contenido político.

En esta investigación que se aborda desde el enfoque crítico no es posible eludir el contexto socio-económico y cultural. Como indica Lozano $^{22}$ (2007), estudiar aspectos comunicacionales desde la teoría crítica implica analizarlos dentro del amplio contexto social, cuestionar su rol en la desigualdad y demanda del/la investigador/a compromiso con el cambio social. Este trabajo no desvincula el análisis del gran contexto en el que se enmarca el objeto de estudio y considera las influencias y determinaciones del modelo económico-político neoliberal, así como los alcances de la globalización y sus efectos sobre los Estados, las sociedades y la cultura.

\section{Metodología}

La investigación tiene carácter no experimental, descriptivo, de tipo transversal, por tanto caracteriza el objeto de estudio y distingue sus interrelaciones con instancias y elementos que nos permiten comprenderlo en su complejidad, ya que se ha realizado bajo el enfoque histórico-crítico.

La investigación está dentro del paradigma mixto cuali-cuantitativo, utilizando entrevistas en profundidad, grupos focales, observación participante y encuesta. Se ha recurrido a una muestra no probabilística, eligiéndola la muestra por casos típicos y casos homogéneos, siguiendo los criterios de pertinencia y adecuación. Las unidades de análisis seleccionadas las conforman la organización no gubernamental denominada Círculo Solidario, 40 mujeres que integran las juntas directivas de organizaciones sociales de base ubicadas en la zona rural

$\overline{22}$ LOZANO, C. (2007). Teoría e investigación de la comunicación de masas. México: Pearson Educación. 
del distrito de Laredo en la provincia de Trujillo (Perú) y el gobierno local de Laredo.

\section{Resultados}

\section{ONG, gobierno local y mujeres de OSB: una relación de verticalidad}

\section{¿Quiénes son las mujeres de OSB y cómo se establece esta relación?}

Las mujeres de OSB pertenecen a sectores empobrecidos e históricamente excluidos de posibilidades de desarrollo. Agrava esta realidad su condición de género por las que han sido subordinadas, debido al modelo patriarcal de sociedad, y reducidas a roles domésticos en el ámbito privado. Situaciones que generan una estructura social y cultural doblemente excluyente para ellas.

Las mujeres que constituyen las unidades de análisis de esta investigación son madres de familia, que no han tenido la oportunidad de profesionalizarse, algunas ni siquiera han terminado la educación básica, y la mayoría de ellas están excluidas de posibilidades laborales por falta de preparación y por su asignación exclusiva de roles domésticos. Pertenecen a las comunidades rurales de Cerro Blanco, Quirihuac, Bello Horizonte y Santa Rosa del distrito trujillano de Laredo. Se eligieron a OSB de estos lugares por haber estado involucradas en proyectos de la ONG y haber sido contactadas por el Gobierno local, de este modo cumplen las condiciones para observar la relación entre las tres instituciones.

En cuanto al aspecto educativo formal, tenemos que el $60 \%$ de las mujeres que tienen cargos dirigenciales en las OSB no han concluido su educación básica regular, un 30\% sí la ha terminado y un reducido $10 \%$ ha tenido oportunidad de estudiar una carrera técnica. Mientras que el $54 \%$ de mujeres (13) que no culminaron sus estudios fue porque sus familias no consideraban importante su educación, el 8\% (2) por decisión propia y un 38\% (9) porque formaron su familia muy jóvenes.

Esta situación las ubica en una condición de desventaja y subordinación ya que su actividad se limita al ámbito doméstico y al amparo económico de su pareja. 
La expresión de machismo no es ajena a su sistema cultural. Se manifiesta de manera explícita e implícita, afectándolas directamente. Los testimonios recogidos lo confirman:

"Hay socias que les gusta asistir a las charlas, pero no vienen muy seguido porque a sus maridos no les gusta, creen que van a hacer otras cosas ¿qué pensarán? Pero algunos sí las dejan, saben que acá estamos puras mujeres, y las que tienen sus hijos chicos los traen a las reuniones del club". Isabel Romero, presidenta de la OSB Santa Rosa de Lima. (Grupo focal en el sector Bello Horizonte).

En la dimensión política no se observa actividad político-partidaria en las comunidades de estas OSB ni compromiso de este tipo en las mujeres; los partidos políticos no tienen presencia orgánica ni la confianza de las señoras. No han tenido experiencia de militancia partidaria; en los casos más cercanos, se ha tratado de simpatías hacia un partido por tradición familiar.

Su cotidianidad está marcada por actividades mayoritariamente domésticas, con algunos matices esporádicos en su organización. $\mathrm{Su}$ vida diaria gira en torno a preocupaciones domésticas, que se aprecia al observar su discurso, en el cual predominan los temas familiares o vecinales. No se ha identificado un discurso con significación política ni con conciencia de género.

Las mujeres se organizan en torno a las OSB porque les significa un beneficio directo:

"el club nos ayuda con el vaso de leche, ya es una ayuda porque no hay plata, muchas familias lo necesitan" y participar en el club es surgir, obtener logros", comentaron en el grupo focal con la OSB Pilar Nores de Bello Horizonte.

La principal motivación para su organización es el brindar apoyo alimentario y como aspiración la implementación de una actividad económico-productiva para las socias. Esta motivación económica está relacionada con los fines asistenciales de su organización. 
"Queremos contar con un taller de costura o con taller de repostería para que tengamos ingresos y podamos dar una mejor atención en nuestro club, para tanto niño que lo necesita", manifestó Neri Vásquez Casamayor, primera vocal del club de madres Pilar Nores de Bello Horizonte.

El tipo de gestión más común que realizan es el de solicitudes de donaciones, implementación de sus locales o mejoras materiales para su organización. Lo que las ubica en una posición de beneficiarias pasivas frente al gobierno local y a la ONG, principales instituciones ante las que canalizan sus peticiones. Las OSB desprovistas buscan un proveedor para estar mejor.

Diversos factores debilitan la capacidad organizativa y articuladora de las OSB, tales como: la relación asistencialista con un Estado deficiente que profundiza la relación de clientelaje, las urgencias de una vida económicamente sufrida que promueve un estilo de vida de supervivencia, los patrones culturales que limitan su incorporación activa en los espacios públicos, el sistema consumista y el individualismo que desarraiga valores como la cooperación y el bien común; provocando la despersonalización y despolitización de sus socias, promoviendo una práctica "light" de ciudadanía.

Las mujeres de las OSB en estudio no evidencian autonomía suficiente para conducir procesos que vayan más allá de la asistencia alimentaria. $\mathrm{Su}$ comportamiento en relación a la ONG es de beneficiarias de proyectos de "desarrollo". Respecto al Gobierno local, la situación no dista mucho, los trámites que realizan son aquellos que persiguen un apoyo concreto, sea para "gestionar la construcción de una vereda a fuera del club" (como el caso del Club de madres de cerro Blanco), para "la implementación de la cocina" (en el Club de madres de Santa Rosa) o para "colocar las rejas de seguridad en las ventanas" (en el Club de madres de Quirihuac).

\section{¿Y cómo se establece la relación entre las OSB, la ONG y el GL?}

La relación se basa en un interés mutuo. Por parte de las OSB se busca la ayuda de la ONG con la finalidad de recibir aportes económicos 
que mejoren las condiciones de su organización. En primera instancia piensan en el apoyo material (mejoramiento y ampliación de infraestructura, implementación, equipamiento de una microempresa, etc.) y como contraprestación participan de las actividades que la ONG diseña en el proyecto, tales como talleres de capacitación. Respecto a la relación con el GL, esta se encuentra fuertemente influenciada por una cultura asistencialista que afianzó y alentó el gobierno populista de Alberto Fujimori, quien no dudó en usar a las OSB con fines proselitistas, estableciendo una relación clientelista, condicionando la entrega de alimentos por el apoyo al gobierno.

La relación descrita se establece en dos sentidos de verticalidad: a) De abajo-arriba, cuando las socias de las OSB asumen la condición de beneficiarias y piden apoyo a instituciones gubernamentales y no gubernamentales. b) De arriba-abajo, en tanto la ONG y el GL recurren a ellas para justificar su trabajo en el primer caso, y para mantener una buena relación con las OSB que asegure contar con mayor respaldo político en los sectores más humildes, en el segundo caso.

\section{La verticalidad discursiva: comportamiento y relaciones internas/externas}

No se produce un encuentro comunicativo, una puesta en común y en horizontalidad de posiciones para crear y recrear nuevos sentidos. En el caso de esta investigación se observa la discursividad vertical que la ONG y el GL estudiados mantienen con las OSB en la relación establecida con la finalidad de promover participación ciudadana de las integrantes de estas organizaciones.

Para entender mejor el análisis de la discursividad vertical es necesario recordar la crítica realizada por Freire (1968) a la forma mecánica en la que se realizaban los procesos de alfabetización. En la visión tradicional se entendía al analfabetismo como un reflejo de la apatía e incapacidad de la gente, hasta que la crítica de Freire la seńala como reflejo de estructuradas de dominación, implantadas en la sociedad y reproducidas por las élites dominantes. Por lo tanto, no podía concebirse una alfabetización auténtica mediante el "depósito" de palabras "vaciadas de 
contenido", es decir, palabras sin praxis, "desprovista de toda relación con el mundo de ese hombre, con las cosas que nombra, con la acción que despliega" (Barreiro en Freire, 2007:9).

Si bien Freire se refiere a la alfabetización, engloba en ella a la toma de conciencia (concienciación) y a la praxis transformadora, lo que sirve para hacer un paralelo de la práctica promotora que realizan la ONG y el GL con la práctica de educación tradicional y bancaria a la que el autor recusa y supera. Este aspecto que se explica con más amplitud en los siguientes ítems, es el que se refiere a lo que hemos llamado verticalidad interna y que se representa en el modelo de verticalidad discursiva.

Empero la verticalidad no se manifiesta solo en una relación interna (entre la ONG-GL y las OSB) sino también se da a nivel externo, tal como se muestra en la figura 02. Ahí vemos cómo la ONG y el GL no construyen autónomamente sus agendas en función de las necesidades de cambio del ámbito en el que intervienen, sino que están condicionadas por instancias externas que regulan sus acciones.

En el caso del GL, a pesar de ser un órgano autónomo, está supeditado a políticas regionales y nacionales con las que se articula, las mismas que son influenciadas por las condiciones en las que se mueve el Estado peruano en este contexto de capitalismo globalizado. La globalización ha afectado la autonomía de los Estados, especialmente de aquellos que se han sometido a los imperativos de la economía transnacional. AnderEgg (2001) afirma que la crisis de operatividad y de legitimad de los Estado-nación han ido estrechando los márgenes que hacen posible la soberanía nacional y la capacidad operativa de los gobiernos.

El Estado peruano se ha adaptado funcionalmente al neoliberalismo transnacional, desde el 2000 ha habido continuidad económica ajustada, aplicadamente, a las reglas transnacionales. En este contexto en que el "mercado" gobierna y el "gobierno" gestiona (Ander-Egg, 2001:48), se comprenden mejor las políticas públicas (nacionales, regionales y locales) y su orientación a cuidar del status quo o en el mejor de los casos a promover un mejoramiento funcional. Entendiendo este marco de influencia exterior y su impacto en lo local, queda más clara la posición del GL de no hacer un esfuerzo mayor que implique un proceso de concienciación, no sólo con las mujeres sino con toda la comunidad, especialmente con los más excluidos. 
La verticalidad externa que alcanza a la ONG proviene de organismos de cooperación internacional y de sus agendas. Las ONG locales que dependen única o mayoritariamente de los fondos de cooperación tienen que adaptar sus líneas de intervención a las demandas de éstos. Martínez $^{23}$ (2011), en una revisión autocrítica del papel de las ONG, da cuenta de su adaptabilidad sorprendente, por un lado a las orientaciones dictadas por la disponibilidad de financiación pública, y por otro lado a la asunción de herramientas (...) que homogenizan la administración de sus trabajos, sin que haya primado reflexión alguna sobre en qué medida el instrumental adquirido y desarrollado contribuye en mayor medida a favorecer procesos de cambio social en el sentido deseado. (2011:8).

Esta característica evidencia que muchas de las ONG implementan sus proyectos de "desarrollo" considerando las indicaciones del cooperante antes que seguir un camino consensuado con la comunidad. Del mismo modo, Martínez cuestiona que las ONG no adoptan una posición política clara por cuidar la "neutralidad" que frente a temas tan delicados como la pobreza no tiene lugar. En este sentido manifiesta:

No se puede seguir hablando de luchar contra la pobreza sin hablar de luchar contra la riqueza. No se puede seguir hablando de procesos de desarrollo sin denunciar la insostenibilidad e irracionalidad del modelo de desarrollo predominante, basado en el crecimiento económico sin contemplar las externalidades. (Martínez 2011: 12).

A eso agregaríamos que la ONG no puede pretender generar ciudadanía en las mujeres de las OSB sin promover espacios de reflexiónacción en los que comprendan las relaciones de poder que existen en el mundo y en los que se recreen su propia visión del mundo y se recojan sus sentidos y expectativas. El trabajo de la ONG Círculo Solidario no cumple con estos criterios de actuación y se limita al cumplimiento de actividades superficiales, aisladas y de poco impacto.

${ }^{23}$ Coordinador de Plataforma 2015 y más, un espacio de ONG e instituciones que velan por el cumplimiento de los objetivos del milenio. 
Figura 02: Niveles de la relación vertical

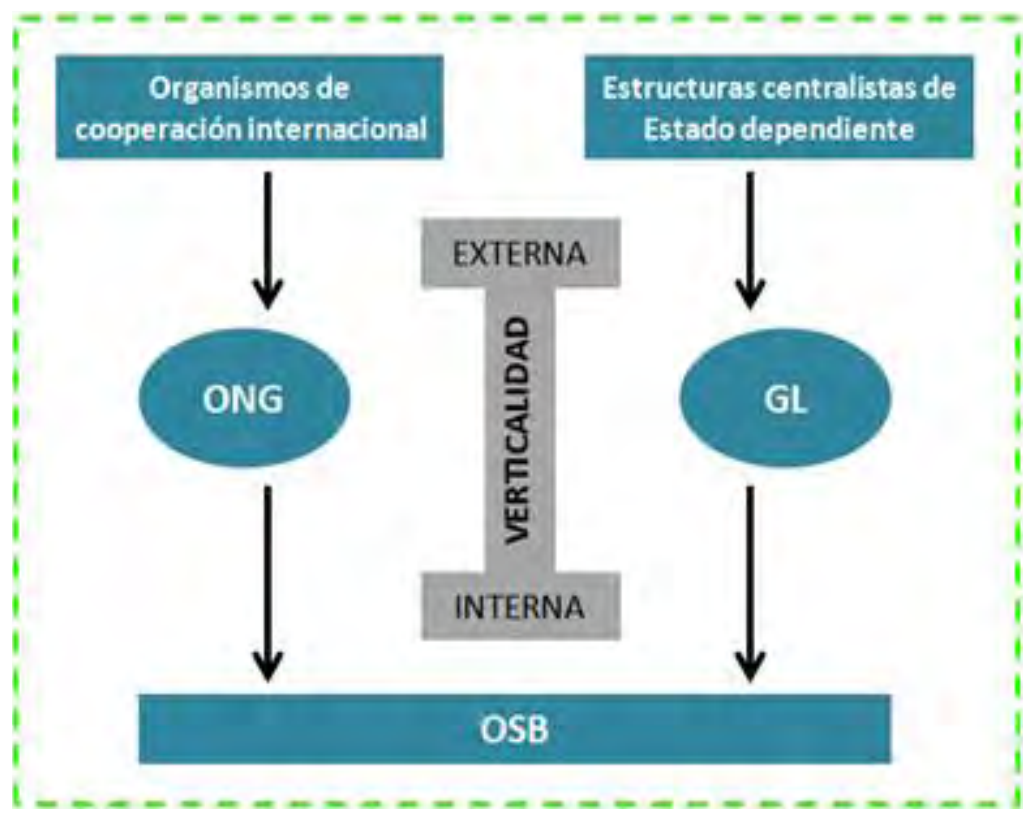

Verticalidad discursiva del GL

Se ha observado el discurso del GL dirigido a las mujeres de las OSB con la finalidad de incorporarlas al mecanismo formal del Presupuesto Participativo (PP). En esta relación se aprecia una supuesta horizontalidad del municipio que "habla" como promoviendo democracia a través de la convocatoria a este espacio participativo. Sin embargo, sus acciones se reducen al cumplimiento de la normatividad presupuestaria del Estado exigida a las municipalidades en los procesos del PP, limitándose a una práctica difusora de estos espacios, sin asumir una posición comprometida con la formación ciudadana, en particular en su vinculación con las mujeres de OSB.

Se aprecia incongruencia entre el discurso declarativo del gobierno local sobre la democracia participativa y su discurso en tanto práctica social, al no atender necesidades comprensivas y de formación en valores cívico-democráticos y de concienciación de las mujeres de OSB que conlleven a una ciudadanía consciente, empoderada y participativa. 
La metodología de la difusión del discurso del GL es típicamente burocrática y vertical, encasillada en esquemas formales y encomendada a operadores administrativos que se limitan a informar los plazos y las fases del proceso y transmiten información general del proceso. Es decir no existe un trabajo articulado, desde una concepción de comunicación horizontal que fomente diálogo problematizador y el desarrollo de pensamiento crítico.

Otro elemento importante que se toma en cuenta en el comportamiento de los emisores, especialmente del GL, es la práctica reproduccionista (entendido este concepto en la propuesta de Louis Althusser, 1976). El filósofo francés manifiesta que el Estado reproduce el status quo de la sociedad a fin de garantizar los privilegios de las élites de dominio, para ello reproducen no solo los medios y formas de producción sino también una reproducción de su sumisión a las reglas de un orden establecido, es decir, una reproducción de sumisión a la ideología dominante.

Althusser, desde su posición marxista, considera que la reproducción del orden social se origina en el plano económico, político y cultural. Este último reproduce la ideología dominante y es lo que nos concierne. Bórquez (2006:114), recoge la lista de los Aparatos Ideológicos de Estado de Althusser: religioso, escolar, familiar, jurídico, político, sindical, de la información (medios masivos) y cultural.

En este caso el GL, como parte de la estructura del Estado, se convierte en un aparato ideológico ${ }^{24}$ con capacidad de intervenir en el campo político estableciendo políticas públicas locales y conduciendo las riendas del Estado en su zona de influencia, en el campo cultural promoviendo o restringiendo determinadas actividades culturales, en el plano familiar por su incidencia directa y cercana en la comunidad, en el plano educativo por las responsabilidades que le han sido delegadas en este campo. La influencia ideológica del GL en las OSB - y más directamente - en las mujeres, se manifiesta cuando no asume una posición de reivindicación de su condición social, cuando continúa

$\overline{24}$ Althusser recupera de Marx y Engels su concepto de ideología como falsa conciencia. Considera que las ideologías se manifiestan de manera práctica, a través de gestos, rituales, conductas y actitudes en general. (Bórquez, 2006:114). 
reproduciendo la relación de dependencia de estas organizaciones a las decisiones que tome la autoridad local, cuando no se implementan políticas en busca de su independencia económica y superación de la subordinación machista que enfrentan, cuando no se les reconoce como ciudadanas en su dimensión política, ni se promueven acciones para su concienciación, actoras capaces de movilizar cambios.

En conclusión, se demuestra la verticalidad discursiva del GL, reproduciendo las condiciones de subordinación en las que se encuentran las mujeres rurales pobres.

\section{Verticalidad discursiva de la ONG}

El discurso de la ONG establecido con las mujeres de OSB es vertical porque está construido con conceptos, propósitos y valoraciones producidos en el escenario tecnocrático de la ONG y colocados por sus agentes de promoción a través de sus proyectos de desarrollo en los cuales las mujeres a las que se dirigen tienen poco que ver.

La verticalidad discursiva carece de una concepción política, teórica y metodológica que resulte como producto de un proceso reflexivo y participativo respecto al tema de la democracia y la participación de las mujeres. Sin su protagonismo no habrá horizontalidad. En consecuencia, es un discurso vaciado de significación política que se va configurando a partir de las orientaciones de la cooperación internacional que financia proyectos de gobernabilidad democrática (según sus estándares de gobernabilidad y de democracia) y de los criterios individuales de promotores de la institución y de profesionales externos contratados para la realización de "capacitaciones".

El comportamiento discursivo es vertical, no se conforman procesos de diálogos reflexivos que permitan el encuentro de las mujeres con su palabra verdadera, esa que nadie se las podrá dar, sino ellas mismas - como lo enseñó Freire - en la unión inquebrantable entre acción y reflexión, es decir en la praxis. Por ello, decir la palabra verdadera sea transformar el mundo (Freire, 2005).

La forma en la que muchas de estas ONG actúan preocupa, ya que se arrogan la lucha contra la pobreza, el fortalecimiento de la gobernabilidad, 
la equidad de género, entre otras proezas, pero al someter al análisis sus prácticas, no llegan a establecer procesos básicos de diálogo con las poblaciones "beneficiarias", por el contrario, predominan los discursos cosméticos y las prácticas superficiales, sin concepción ni posición política. Este rol puede encasillar a estas ${ }^{25} \mathrm{ONG}$ como instituciones de contención social.

\section{Participación crítica de resistencia comunicativa frente a la participación persuasivo-adaptativa}

De la verticalidad discursiva explicada en los ítems anteriores se espera que haya una respuesta de las receptoras, esta se configura en una participación persuasivo-adaptativa, esto es una participación ¡“de ficción”! (Barbero, 2003:28), desconectada de una praxis liberadora que se adecúa a las tesis reproduccionistas de las que nos advierte Althusser (1976).

Frente a este enfoque discursivo se propone la horizontalidad comunicativa como alternativa, aquella que está perdiendo espacio en las formaciones universitarias de comunicación y cuyas tesis de distintos autores confluyen en propuestas críticas, liberadoras y humanizantes. Sólo de la comunicación podemos esperar una participación sustantiva, organizada y consciente, es decir una resistencia comunicativa que construya en el seno de la horizontalidad marginal, esto es, al interior de los vastos sectores populares nuevas condiciones materiales y espirituales de existencia.

\section{Conclusiones}

1. La verticalidad discursiva del GL se hace tangible en tanto se limita al cumplimiento adjetivo de la normatividad presupuestaria del Estado, condicionada a su vez por una relación vertical

$\overline{{ }^{25} \text { Nos referimos a }}$ las organizaciones que tienen estas características y no realizan un trabajo político real de cambio social, no se incluye en esta generalización a las ONG que sí cumplen procesos comunicativos emancipadores. 
externa de las estructuras centralistas de un Estado dependiente que reproduce las condiciones de subordinación en las que se encuentran las mujeres rurales pobres.

2. La verticalidad discursiva de la ONG se evidencia en tanto carece de una concepción política, teórica y metodológica propia. Su discursividad está orientada por una verticalidad externa a partir de las consideraciones de los organismos de cooperación internacional.

3. No se observa en las integrantes de las OSB una actuación autónoma y de propia iniciativa que refleje un ejercicio ciudadano comprometido con su desarrollo. Su participación es orientada y de motivación asistencial, no cuestiona el sistema establecido ni su condición de exclusión, contribuyendo así al reforzamiento del statu quo. La relación con la ONG y el GL no se refleja en un empoderamiento ni autonomía suficiente.

4. Se constata que en la relación de las mujeres de OSB tanto con la ONG como con el GL no existe un trabajo articulado, desde una concepción de comunicación horizontal que fomente diálogo problematizador y el desarrollo de pensamiento crítico. Se observa en su lugar eslabones verticales de comunicación.

5. Existe distancia cultural entre los emisores del discurso y las receptoras. La dinámica cultural y socializadora de las mujeres de OSB está alejada de espacios políticos y de requerimiento institucional, $y$, claramente comprometida con prácticas comunitarias en torno a motivaciones cotidianas. 


\section{$\underline{\text { Referencias }}$}

Althusser, L. (1976). Ideología y aparatos ideológicos de Estado. En Revista Mexicana de Ciencia Política, número 78, año XX. México, UNAM. Ander-Egg, E. (2001). Globalización: El proceso en el que estamos metidos. Argentina: Editorial Brujas.

Barreiro, J. (1974). Educación popular y proceso de concientización. Argentina. Siglo Veintiuno Editores.

Bórquez, R. (2006). Pedagogía Critica. Editorial Trillas. México.

Freire, P. (1968). La alfabetización de adultos. Critica de su visión ingenua, comprensión de su visión critica, en Cristianismo y sociedad. Montevideo. S/E.

Freire, P. (2005). Pedagogía del oprimido. México. Siglo Veintiuno Editores.

Freire, P. (2007). La educación como práctica de libertad. México. Siglo Veintiuno Editores.

Lozano, J. (2007). Teoría e investigación de la comunicación de masas. México: Pearson Educación.

Martín-Barbero, J. (2003). La educación desde la comunicación. Colombia. Grupo Editorial Norma.

Martínez, P. (2011). Redefinición del papel de las ONG. http:// www.2015ymas.org/IMG/pdf/Pablo_Martinez_Oses.pdf 